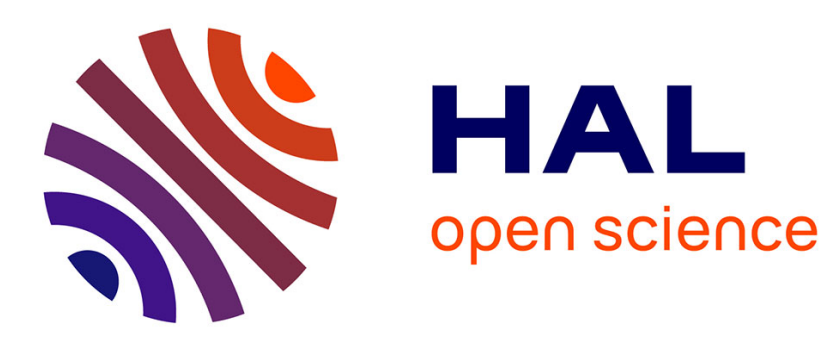

\title{
The Representation of Time in Discrete Mechanics
}

Vincent Ardourel, Anouk Barberousse

\section{To cite this version:}

Vincent Ardourel, Anouk Barberousse. The Representation of Time in Discrete Mechanics. Time of Nature and the Nature of Time, , p. 173-208, 2017, 978-3-319-53723-8. hal-01946028

\section{HAL Id: hal-01946028 \\ https://hal.science/hal-01946028}

Submitted on 5 Dec 2018

HAL is a multi-disciplinary open access archive for the deposit and dissemination of scientific research documents, whether they are published or not. The documents may come from teaching and research institutions in France or abroad, or from public or private research centers.
L'archive ouverte pluridisciplinaire HAL, est destinée au dépôt et à la diffusion de documents scientifiques de niveau recherche, publiés ou non, émanant des établissements d'enseignement et de recherche français ou étrangers, des laboratoires publics ou privés. 


\title{
The Representation of Time in Discrete Mechanics
}

\author{
Vincent Ardourel \& Anouk Barberousse
}

Received: date / Accepted: date

\section{Introduction}

As an important innovation of the last decades, there exists nowadays an intrinsically discrete physical theory called Discrete Mechanics (DM). It is intrinsically discrete since it does not originate in the discretization of continuous equations but is rather built upon already discrete fundamental principles. DM is a new, variational theory of classical motion. Our leading assumption in examining the implications of DM for the representation of time is that DM is a "good" physical theory, at least as good as our best physical theories for all practical purposes, and even better, because it allows for easier and more precise predictions due to its straightforward implementation on computers. This assumption will be argued for below in the paper.

The purpose of the paper is to examine the consequences of adopting of this discrete physical theory on the way we can represent time in physics. We focus on two main consequences. The first is the use of a discrete representation of time, the second is the representation of time as a dynamical variable rather than a parameter. In his seminal paper on DM, the Nobel Prize winner Tsung Dao Lee raises the following questions:

In place of treating time as a continuous parameter, we may ask: (1) Can time be a discrete parameter (discrete time formulation)? (2) Can time be discrete and treated as a bona fide dynamical variable (discrete mechanics)? (Lee 1983, p. 217)

We take on Lee's questions and analyze the implications of positive answers to both.

In the first section of the paper, we give a brief presentation of DM, emphasizing that it can be considered a genuine physical theory, not merely a numerical method. In section 2, we argue that DM points out that the continuous representation of time is dispensable and discuss the implications of this claim. In section 3, we explain why the time symbol is a dynamical variable in DM and present some implications of this original feature of DM.

Université Lille 1 \& UMR Savoirs, Textes, Langage 


\section{Discrete Mechanics}

In order to answer the question we are interested in about the implications of using a discrete representation for time, we first present in some details the way scientists introduce discrete representations of time in their models. In section 1.1, we review various discretization practices and in section 1.2, we focus on the recent technique of variational integrators, which leads us to present Discrete Mechanics, the theory we are going to analyze further in the remaining of the paper. Finally, in section 1.3, we make clear that DM is not merely a numerical technique but has to be viewed as a discrete physical theory or, at least, as a discrete formulation of classical mechanics.

\subsection{Discretizing time in computer models}

All attempts at building up a discrete theory of mechanics originate in the computer era, that is, in a context in which discrete models proliferate. In order to better capture the specificity of DM, it is useful to first present how time and other magnitudes are discretized in order to benefit from the machines' computational power.

For sure, computers are a tremendous help for solving the equations given by the physical theories. However, computational power has a cost, as using computers requires to transform the continuous equations into discrete ones because they can only deal with discrete values. As a result, contemporary modeling practice is throughout defined by the need for designing algorithms to transform continuous equations into discrete ones that can be solved by computer algorithms. In this section, we first present some common types of numerical integrators and we compare their respective merits, focusing on the more efficient ones, the variational integrators.

\subsubsection{Types of integrators}

The transformation of continuous equations into discrete ones is obtained by numerical integrators. Among the different numerical integrators, we focus on the simplest ones: the forward/explicit Euler integrator and the backward/implicit Euler integrator. The first numerical integrator transforms the time derivative $d x / d t=y$ into the discrete equation $\left(x_{k+1}-x_{k}\right) / h=y_{k}$. With the second integrator, the time derivative $d x / d t=y$ is transformed into the discrete equation $\left(x_{k+1}-x_{k}\right) / h=y_{k+1}$.

Consider, as an example, the motion equations of a simple pendulum solved with these integrators. Two differential equations have to be transformed. The first one represents the definition of the angular speed $d q / d t=v$, with q is the pendulum's angle with the vertical. The second equation represents the evolution of the angular speed with respect to the size of the pendulum 1 and the acceleration of gravity $g$ : $d v / d t=-g / l \sin (q)$. For each integrator, both differential equations are transformed into discrete equations according to the previous rules. With the forward Euler integrator, the differential equations are transformed into the discrete equations:

$$
\left(q_{k+1}-q_{k}\right) / h=v_{k} \text { and }\left(v_{k+1}-v_{k}\right) / h=-g / l \sin \left(q_{k}\right)
$$

With the backward Euler integrator, they are transformed into: 

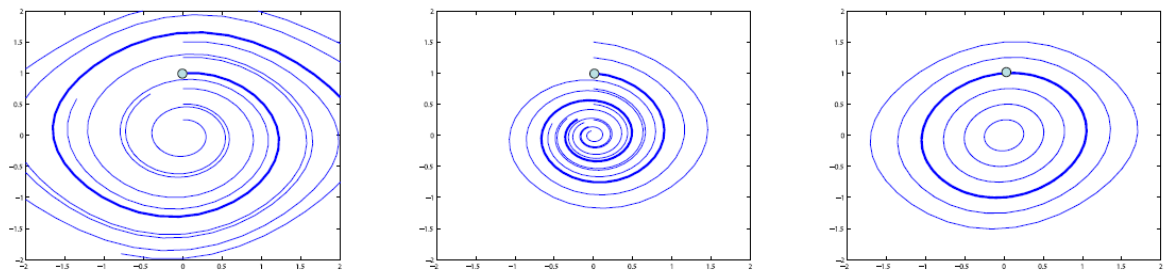

Fig. 1 The simple pendulum in the phase space $(q, v)$ solved with three Euler integrators: forward Euler (left), backward Euler (middle), symplectic Euler (right). For each integrator, there are six different trajectories that correspond to different initial conditions. The bold lines in each of the three figures correspond to the same initial condition. (Stern \& Desbrun 2008, p. 77)

$$
\left(q_{k+1}-q_{k}\right) / h=v_{k+1} \text { and }\left(v_{k+1}-v_{k}\right) / h=-g / l \sin \left(q_{k+1}\right)
$$

Let us introduce another numerical integrator to solve the simple pendulum: the variational/symplectic Euler integrator. It is a mix of the first two Euler integrators since the first differential equation is solved with the backward integrator and the second equation, with the forward Euler integrator. In this case, the differential equations are changed into:

$$
\left(q_{k+1}-q_{k}\right) / h=v_{k+1} \text { and }\left(v_{k+1}-v_{k}\right) / h=-g / l \sin \left(q_{k}\right)
$$

\subsubsection{Comparison}

Numerical tests of have been performed by Stern and Desbrun (2008) in order to compare the three Euler integrators. The result of the comparison is presented in Figure 1, in which the phase space trajectories of the pendulum are computed for six different initial conditions for each integrator. Continuous Newtonian mechanics requires that the trajectories of the pendulum be closed curves since it is a conservative system. However, as we can see on the left, the trajectories obtained with the forward Euler integrator are increasing spirals due numerical instability. With the backward Euler integrator (in the middle), on the contrary, the trajectories are decreasing spirals due to numerical dissipation. Only the symplectic Euler integrator (on the right) exhibits the right behavior: the trajectories are closed curves, which means that the energy of the pendulum is conserved.

Hairer et al. (2002) performed similar numerical tests for the computed trajectories of the planets of a reduced solar system. Numerical computations of the motion of the five outer planets relative to the Sun, over a time period of 200000 days, are carried out with the forward, backward and symplectic Euler integrators (Figure 2). They lead to similar behaviours. With the forward integrator, the trajectories are instable: the planets leave their stable orbits. With the backward integrator, the trajectories are not even periodic: the planets collapse on the sun.

As shown in the figures, variational integrators generally give better results than traditional ones. The main reason for this difference is that most traditional algorithms are non-symplectic: they do not preserve the geometric properties of the system's dynamics. This is especially inconvenient when the represented systems are 


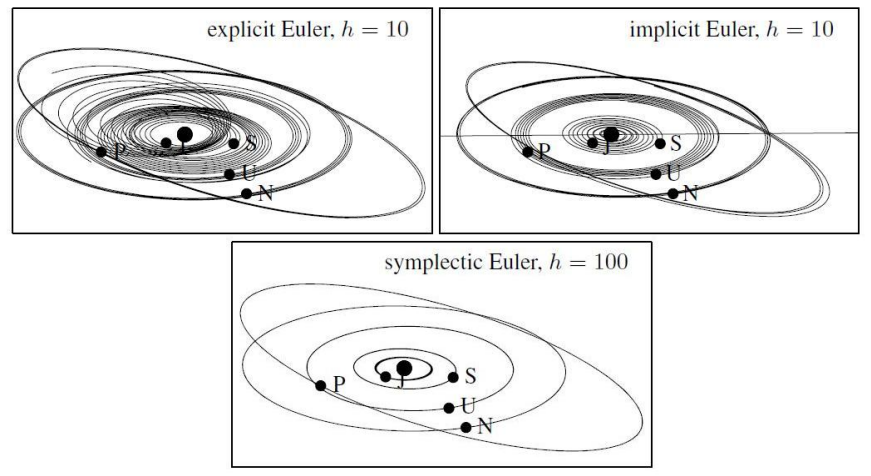

Fig. 2 The trajectories of the planets of a reduced solar system computed with three Euler integrators: forward Euler (at the top left), backward Euler (at the top right), symplectic Euler (below). A 10 day time step is used with the forward and backward Euler integrators while a 100 day step is used with the symplectic Euler. For each integrator, the initial conditions correspond to the positions of planets on September 5, 1994 at 0h00 (Hairer et al. 2002, p. 14).

conservative. In this case, the problem of the traditional algorithms is that they cannot be conservative unless they include artificial mechanisms guaranteing a not-tooincorrect resulting behavior. However, introducing a mechanism of this type is bound to distort the results. By constrast, variational integrators are symplectic: they naturally preserve the global geometry of the system's dynamics and do not need any artificial, parasitic mechanism to remain stable.

\subsubsection{The value of variational integrators}

Using traditional algorithms requires one to apply the following strategy: first, write down local, specific equations and second, discretize these specific equations. The price of this strategy is the risk of introducing numerical artefacts that are sometimes necessary to guarantee correct global properties. By contrast, using variational integrators allows one to focus on the correct representation of global structures and invariants. Kang Feng, who has been a major contributor to the field of variational integration, realized in the 1970's that the choice between the two strategies has major consequences. As his brother emphasizes in the foreword of a Kang Feng's posthumous book:

[Kang Feng] fully realized that different mathematical expressions for the same physical law, which are physically equivalent, can perform different functions in scientific computing [...]. In this way, for classical mechanics, Newton's equations, Lagrangian equations and Hamiltonian equations will show a different pattern of calculations after discretization. Because the Hamiltonian formulation has a symplectic structure, he was keenly aware that, if the algorithm can maintain the geometric symmetry of symplecticity, it will be possible to avoid the flaw of artificial dissipation of this type of algorithm and design a high-fidelity algorithm. (Kang Feng and Mengzhao Qin 2010, p. x) 
At first view, the difference between traditional and variational algorithms might appear a purely technical one, only relevant to applied mathematics. However, this judgment would be a reductive conception of the field of discretized models. The development of this field has indeed given rise to researches that are not confined to applied mathematics but also pertain to physical theory. We present these recent advances in the next section.

\subsection{Variational integrators and Discrete Mechanics}

Discrete Mechanics, which is our main focus in this paper, originates in two research trends. The first one is based on the need to design discrete integration algorithms, and the second one is what we shall call the "geometrical turn" of physical theories. Since the second half of the twentieth century, modern geometrical formulations of Lagrangian and Hamiltonian mechanics have been developed and, nowadays, differential geometry is the "mother tongue" of physical theories (Butterfield 2006a; 2006b). As a result, it is common to consider the geometric structure of the investigated systems as very important. This is the reason why discrete models preserving this geometric structure are now favored over other models. As Stern and Desbrun have it,

the very essence of a mechanical system is characterized by its symmetries and invariants. Thus preserving these symmetries and invariants (e.g., certain momenta) into the discrete computational setting is of paramount importance if one wants discrete time integration to properly capture the underlying continuous motion. (Stern \& Desbrun 2008, p. 75)

Discrete mechanics results from the will to respect the global geometrical of mechanical behaviors and from the enhanced power of the induced integrators 1

Under its Lagrangian form, classical mechanics can be treated from a geometrical point of view as it is grounded on a variational principle, the principle of least action. In Lagrangian mechanics, the trajectories of bodies are viewed as geodesic in a state space. Discrete mechanics also adopts a geometrical point of view to describe mechanical behaviors as it is but a discrete version of Lagrangian mechanics. Consequently, the link between variational integrators and discrete mechanics is strong, as emphasized by Stern and Desbrun in the following quote:

The driving idea behind discrete geometric mechanics is to leverage the variational nature of mechanics and to preserve this variational structure in the discrete setting. [...] [I]f one designs a discrete equivalent of the Lagrangian, then discrete equations of motion can be easily derived from it by paralleling the derivations followed in continuous case. In essence, good numerical

\footnotetext{
1 We would like to emphasize that, at the beginning of the 1980s, Tsung-Dao Lee $(1983,1987)$ developed a Discrete Mechanics for another reason still. It was to solve the well-known divergence problems of Quantum Field Theory. He wanted to build up a Discrete Mechanics conceived as a first step toward fully discrete fundamental theories in which divergences could not occur. The idea was first to try to develop one discrete theory in order to generalize it to others. To our knowledge, it did not go very far toward QFT.
} 
methods will come from discrete analogs to the Euler-Lagrange equations -equations that truly derive from a variational principle. (Stern \& Desbrun 2008, p. 79)

Among the discrete versions of Lagrangian mechanics, some are more or less ad hoc, but DM is anything but ad hoc as its starting point is the discretization of the variational principle itself. Having such a sound foundation is the main virtue of DM since it enables its users to preserve the geometrical properties of variational mechanics. Consequently, the discrete motion equations derived from the discrete principle - the discrete Euler-Lagrange equations - also preserve the geometrical properties of mechanical behaviors. Most importantly, symplecticity and other physical properties like the conservation of momenta are preserved in DM.

In order to make clear how DM is linked with variational integrators, we introduce a few formulae in order to present how the simple pendulum is treated within DM. In the continuous Lagrangian mechanics, the equations of motion are derived from the minimization of the action $S_{c}$. It is defined as the integral over time of the Lagrangian $L_{c}$ of the system: $S_{c}=\int L_{c} d t$. In the case of the simple pendulum, the Lagrangian is:

$$
L_{c}=\frac{1}{2} m l^{2} v^{2}+m g l \cos (q)
$$

with $q$ the pendulum's angle with the vertical, $v=d q / d t$, the angular speed, $m$ the mass of the body, $l$ the length of the pendulum, and $g$ the acceleration of gravity. The minimization of $S_{c}$ leads to the equations of motion introduced in section 1.1.1:

$$
d v / d t=-g / L \sin (q)
$$

Similarly, in DM, the discrete action $S_{d}$ is defined as $S_{d}=\sum L_{d} h$ where $L_{d}$ is the discrete Lagrangian of the system and $S_{d}$ its sum over discrete time ${ }^{2}$ In the case of the simple pendulum, the discrete Lagrangian can be defined as:

$$
L_{d}=1 / 2 m l^{2} v_{k}^{2}+1+m g l \cos \left(q_{k}\right)
$$

Where the pendulum's angle is $q_{k}$ and the angular speed $v_{k+1}=\left(q_{k+1}-q_{k}\right) / h$. The minimization of $S_{d}$ leads to the discrete equation of motion:

$$
\left(v_{k+1}-v_{k}\right) / h=-g / L \sin \left(q_{k}\right)
$$

We now see how DM is linked with variational integrators. Equation (7) and the definition of the discrete angular speed are precisely the discrete equations that correspond to the symplectic Euler integrator introduced in the section 1.1.1.

To sum up, DM is a discrete version of classical mechanics with a discrete least action principle as first principle, from which the discrete motion equations are derived. These discrete equations are very important in a numerical context. They are

\footnotetext{
2 We would like to emphasize that there is no single way to define a discrete Lagrangian for a mechanical system. The choice of another discrete Lagrangian for the simple pendulum leads to a different equation of motion that corresponds to another variational integrator. In other words, it can be admitted that there are as many DMs - or versions of DM - as ways to define discrete Lagrangians. We go back to this point in the conclusion of the paper.
} 
variational integrators that, as we have seen in section 1.1 .2 , lead to very stable computations.

A last point has to be made clear. Two slightly different versions of DM have to be distinguished: a "standard version" and an "extended version". Until now, we have focused on the "standard version". This version requires constant time step $h$. Time is represented as a series of equally spaced instants, $t_{1}, t_{2}, t_{3} \ldots t_{k-1}, t_{k} \ldots$ where $t_{2}-t_{1}=t_{3}-t_{2}=t_{k}-t_{k-1}=\ldots=h$. By contrast, the "extended version" of DM requires a variable time step $h_{k}$. Within this version, the instants $t_{1}, t_{2}, t_{3} \ldots t_{k} \ldots$ are not equally spaced.

The standard version is a particular case of the extended version for which the discrete time step $h_{k}$ is constrained to be constant. This standard version is developed in order to build variational integrators, like the previous symplectic Euler integrator. It allows indeed for efficient numerical computation. The extended version is generally less easy to implement on computers. However, it leads to a more satisfying theory in the sense that it enables for mechanical systems to have more conserved quantities. Like the standard version, the extended version preserves symplecticity and the momenta. But in addition, it preserves energy exactly. J.E. Marsden, one of the major creators of DM, makes clear how to go from the standard version to the general theory:

Unlike the standard discrete variational mechanics [...] however, we extend the framework to include time variations in addition to the usual configuration variable variations, as in Lee (1983) and (1987). [...] [W] obtain an extra equation which exactly ensures preservation of a quantity we can identify as the discrete energy. In this way, both the definition of the discrete energy and the fact that it is preserved arise naturally from the variational principle. (Marsden et al. 2001, p. 463)

As we have seen in this section, energy is reasonably well conserved when the symplectic Euler integrator based on the standard version of DM is used. This is one of the main virtues of such integrators with respect to the traditional ones. However, energy is not exactly conserved within the standard version. Energy oscillates around a mean value. By contrast, within the extended version, energy is exactly conserved. In the remaining of the paper, we focus on the extended version as the fundamental version of DM, the standard version being a simplified version for practical needs.

\subsection{DM as a physical theory}

As should be clear from section 1.2, the principles governing the development of discrete mechanics are very different from the recipes allowing physicists to implement their models on a computer. The strategy is not: write out the equations first, and then find out a way to solve them on the computer, but rather: let us begin with discrete fundamental equations and find out the solutions of the specific equations within this general framework. Therefore, we argue that discrete mechanics is a genuine discrete physical theory rather than a mere set of numerical models or numerical techniques. This claim agrees with the terminology used by one of the first creator of DM, the 
1957 Nobel Prize of Physics T.D Lee, who calls DM a "new theory" (Lee 1987, 844). In this section, we review some reasons why DM can be considered a genuine physical theory.

First, DM possesses an empirical content. Its equations can be interpreted as providing information about physical phenomena. In particular, the discrete EulerLagrange equations can be interpreted as describing the dynamics of mechanical systems. We present below the cases of two elementary physical systems, a falling body subjected to the gravitational potential and a harmonic oscillator (Section 2.3.2). We will see in these examples how DM allows one to represent the temporal evolution of physical systems.

Current developments of DM strongly suggest that DM is empirically adequate as continuous classical mechanics is. In other words, DM and continuous mechanics are empirical equivalent. The first indication of the equivalence is that the equations of DM tend to the equations of continuous mechanics when discrete time steps go to zero (D'Innocenzo et al. 1987, p. 246). Therefore, one can assume that if the discrete time steps are taken sufficiently small, the predictions of DM cannot be distinguished from those of continuous mechanics. We do not have space enough to discuss this point. However, to make this point clear, let us take the example of the harmonic oscillator system. It can be proved that the frequency of the oscillator within DM differs from the frequency within continuous mechanics by a factor $h^{2}$ (D'Innocenzo et al. 1987, p. 250). Consequently, if the time step $\mathrm{h}$ is taken sufficiently small, the frequencies are as close to each other as we want and, thus, cannot be distinguished by any physical measure 3

Secondly, let us focus on the way its theoretical statements are linked to each other. DM has its own first principle: a principle of least discrete action. The laws of DM, like the discrete equations of motion and the discrete law of conservation of energy, are deduced from this first principle. Other properties like the symplecticity of the equations of motion are also deduced form this first principle. As a result, DM is a physical theory from the point of view of its deductive architecture: it is a set of hierarchically ordered theoretical statements.

Thirdly, DM allowing for exact results for some its equations provides another argument in favor of its being a genuine physical theory. For instance, it is possible to exactly solve, without relying on numerical computation, the equations of motion of a harmonic oscillator. As we show in section 2, exact results are important in physical theories because they enable scientists to describe and explain the behavior of elementary physical systems.

Even though DM possesses some important features of physical theories, it is unclear whether it is an independent theory from continuous classical mechanics or rather a formulation thereof. If the latter, DM's first principle and fundamental laws would be discrete versions of the principles and the laws of continuous classical mechanics rather than autonomous statements. The only difference between DM and continuous classical mechanics would be notational and would by no means affect empirical content.

\footnotetext{
3 We emphasize that there is not a unique DM but a family of DM depending on the (initial) value of the discrete time step. Thus, when we claim that DM and continuous are empirically equivalent, we mean more precisely that it exists at least one DM that is empirically equivalent to continuous mechanics.
} 
The claim that DM has the same empirical content as continuous classical mechanics but is differently formulated is consistent with the way DM is used in physics. The main use of DM is indeed the numerical study of the behavior of mechanical systems. Consequently, contributors to the development of DM define it explicitly as "a formulation ${ }^{4}$ of mechanics in discrete-time that is based on a discrete analogue of Hamilton's principle, which states that the system takes a trajectory for which the action integral is stationary" (Lee et al. 2009, p. 2001). But DM being a formulation of classical mechanics rather than an independent theory of classical motion does not mean that it does not call for analysis. On the contrary, the existence of different, however equivalent, formulations of a same theory calls for a specific analysis.

Even though they have the same empirical content, different formulations of the same theory do not say exactly the same about their common domain of phenomena. They describe them with the help of different equations, requiring different concepts. For example, the Newtonian formulation of mechanics requires the concept of force whereas the Hamiltonian formulation requires the concept of energy. Even though this distinction is not empirically relevant, in the sense that no physical measure could make any difference, it may be relevant from a theoretical or metaphysical point of view. Therefore, in order to discuss the metaphysical consequences of a physical theory, attention has to be paid to its formulations.

For instance, in the case of continuous classical mechanics, J. North argues that, even though they are empirical equivalent, Lagrangian and Hamiltonian formulations have "irreconcilable differences":

Lagrangian and Hamiltonian mechanics may be equivalent for the purpose of doing classical mechanics. Nonetheless, there are important differences between them. There are differences in structure. (North 2009, p. 72)

The two formulations are based on different state spaces. The state of a mechanical system is described by the position $q$ and its derivative $d q / d t$ within the Lagrangian formulation while it is described by $q$ and its conjugate momentum $p$ within the Hamiltonian formulation. The state spaces do not have the same mathematical structure: the former is a Riemannian manifold and the latter a symplectic manifold. Even though they are connected by an isomorphism (North 2009, p. 84), the difference is by no means negligible:

Modern geometric formulations of physics suggest that there is more to a theory's empirical content than its set of dynamically possible histories. There is also the statespace in which those histories are traced out. And there is the structure of that space. The equivalence of theories is not just a matter of physically possible histories, but of physically possible histories through a particular statespace structure. Hamiltonian and Lagrangian mechanics are not equivalent in terms of that structure. This means that they are not equivalent, period. (North 2009, p. 79)

The equivalence is thus only empirical, but does not extend to other aspects.

Similarly, the state space of DM, and more precisely the state space of discrete Lagrangian mechanics, is different from the state space of continuous Lagrangian

4 We emphasize. 
mechanics. As there is no velocity variable $d q / d t$ within DM, the state spaces have different structures. In DM, there are only discrete positions at successive instants $q_{k}$ and $q_{k+1}$. The notion of speed is not fundamental; rather, motion is represented by series of discrete positions. Therefore, there are also "irreconcilable differences" between DM and continuous Lagrangian mechanics. The differences might be even more irreconcilable than in the case discussed by North since, as Marsden et al. emphasize, the state space of DM "is not locally isomorphic to the [...](traditional) Lagrangian state space"(2001, p. 473).

To sum up, even if their predictions cannot be distinguished by physical measures, DM and continuous Lagrangian mechanics are not equivalent throughout. In the remaining of the paper, we discuss the implications of the use of DM for the representation of time.

\section{What does it imply to use a discrete representation of time?}

As made explicit in Section 1, the use and development of DM relies on the adoption of a discrete representation of time. In this section, we answer the question: What does it imply to use a discrete representation of time when doing physics, that is, when trying to describe, predict and explain physical phenomena? We study three possible answers to this question in turn. The first tentative answer is that it implies using equations that are not as fundamental as continuous equations. We dismiss this answer. The second answer is that it implies to dispense with the mathematical continuum. We also dismiss this answer and explain why. The last answer we discuss is that it implies that it is possible to dispense with the continuous representation of time in physics. As the meaning of this answer is difficult to capture, we first endeavor to give a precise meaning to the dispensability of the continuous representation of time in physics. Then we give some arguments showing that this answer is highly plausible.

\subsection{Discrete equations and fundamental laws}

It is generally assumed that differential equations, which are continuous, are more fundamental than difference equations, which are discrete. This opinion relies on the belief that difference equations necessarily derive from differential equations. Even though difference equations are pervasive in current physics as well as in other scientific domains, they are commonly considered as inessential substitutes of the continuous equations.

For sure, most of the discretized equations that are used on a day-to-day basis are derived from general, continuous equations associated with a lot of restricting, idealizing, and approximating hypotheses. They are thus very specific to the model at hand. However, as it should be clear from Section 1.2, not all difference equations are model-specific. DM's difference equations are perfectly general equations, as discrete mechanics is not devoted to finding out the solutions to specific-purpose models. Here is the way Lee presents the contrast between differential and difference equations within DM: 
For more than three centuries we have been influenced by the precept that fundamental laws of physics should be expressed in terms of differential equations. Difference equations are always regarded as approximations. Here, I try to explore the opposite: Difference equations are more fundamental, and differential equations are regarded as approximations. (Lee 1987, p. 859)

It is clear from this quote that using a discrete representation of time does not imply using less fundamental equations within DM. In Lee's theory, difference equations are assumed to be the first equations of the theory. Accordingly, differential equations derive from difference equations. They result from a limit procedure on difference equations, when the discrete time steps tend to zero. Thus, while in continuous mechanics differential equations are fundamental and difference equations result from a discretization procedure, in DM difference equations are fundamental and differential equations result from a limit procedure.

\subsection{Discrete equations and mathematical continuum}

Now that we have dismissed the claim according to which using a discrete representation of time necessarily implies using non-fundamental equations, we turn to the question whether using a discrete representation of time implies dispensing with the mathematical continuum altogether. The answer to this question is simple and factual, as DM, although using a discrete representation of time, does not dispense with real numbers. On the contrary, the restriction to rational numbers would amount to a dramatic loss in predictive and explanatory power. In order to argue in favor of these two points, we first show how DM makes use of real numbers. Then, we briefly describe an older version of DM whose purpose was precisely to dispense with the mathematical continuum, in order to make the limits of this previous version clear.

In the version of DM we focus on, the discrete time symbol is defined as $t_{k}=k h_{k}$ where $k$ is a natural number and $k<N$, with $N$ fixed, and the time steps $h_{k}$ are real numbers (Marsden et al. 2001, p. 370); as a result, the "instants" $t_{k}$ are also real numbers. It is important to recall that when a variable takes its value within a discrete set, it is not necessarily rational for all that. The discrete set in question may be composed of real numbers. Thus, the difference between a discrete representation of time and a representation of time by rationals only is best expressed in terms of the cardinality of the sets of instants. In the continuous representation, a time interval is represented by an uncountable infinite set of instants. On contrary, in the discrete representation, no representation of intervals is available, but only representations of sets of time points, represented by discrete sets of real numbers.

Let us complete this factual point about the discrete representation of time not being restricted to rational numbers by pointing out that real numbers play a truly important role within DM for another reason yet: the proof of certain general results in DM actually requires the use of real numbers and mathematical operators defined on real numbers. For instance, a discrete Noether theorem can be proved within DM by relying on real numbers. It is an important theorem since it states that physical quantities are conserved when a mechanical system satisfies some symmetry. More 
precisely, Noether theorem refers to differentiable symmetries of the system's Lagrangian. For instance, if a mechanical system behaves in the same way regardless of where it is located, the system is symmetric under translations in space. By Noether theorem, the linear momentum of the system is conserved. Further, if a system is rotationally symmetric, Noether theorem states that its angular momentum is conserved. As far we know, Noether theorem and its proof, in DM as well as in conventional mechanics, require using differential calculus, and, as such, they rely on real numbers 5

In order to better appreciate the importance of real numbers in DM, we now present the limitations that are inherent to an older version of DM whose project was to precisely dispense with real numbers. It was indeed Donald Greenspan's purpose to develop a discrete physical theory that would appeal to real numbers in none of its descriptions or computations. During the 1970s and 1980s, Greenspan developed what he called a "discrete mechanics" (Greenspan 1973, p. 10) that is different from the theory we focus on in this paper. His discrete mechanics consists in reformulating traditional Newtonian mechanics with a discrete representation of time. In Greenspan's discrete mechanics, time is represented by a discrete parameter tk where $\mathrm{tk}=\mathrm{kh}, \mathrm{k}$ being a natural number and $\mathrm{h}$ a rational number. The differential equation expressing Newton's second law is replaced by a difference equation with a discrete acceleration and a discrete force $m \mathbf{a}_{k}=\mathbf{F}_{k}$. The discrete acceleration $\mathbf{a}_{k}$ is defined as the ratio $\left(\mathbf{v}_{k+1}-\mathbf{v}_{k}\right) / h$, with $\mathbf{r}_{k}$ the discrete velocity vector, and the discrete force $\mathbf{F}_{k}$, as a function of a discrete potential energy. More precisely, the discrete force is written as:

$$
\mathbf{F}_{k}=\frac{V\left(\mathbf{r}_{k+1}\right)-V\left(\mathbf{r}_{k}\right)}{\mathbf{r}_{k+1}-\mathbf{r}_{k}} \frac{\mathbf{r}_{k+1}+\mathbf{r}_{k}}{r_{k+1}+r_{k}}
$$

with $V\left(\mathbf{r}_{k}\right)$ the discrete potential energy and $\mathbf{r}_{k}$ the discrete position vector at the instant $t_{k}$.

Greenspan and LaBudde show that, under few assumptions, all the main conservation laws of the traditional Newtonian classical mechanics can be established within their version of discrete mechanics, for discrete linear momentum, energy and angular momentum are exactly conserved (LaBudde and Greenspan 1974).

For Greenspan and LaBudde, the best argument in favor of their version of a discrete mechanics is that exact conservation of discrete quantities, like energy, allows for more stable numerical computations. Since energy and momenta are constrained to be equal to their initial values, they cannot diverge and thus cannot lead to instable computations. Greenspan argues that the development of his discrete mechanics shows that scientists can study physical phenomena without relying on the mathematical continuum. At the beginning of his book on discrete mechanics, he emphasizes:

The concept of infinity and the consequential concepts of limit, derivative, and integral are reasonable for the pure mathematical study of real numbers and real functions, but are not reasonable for the modeling of physical phenomena. [...]

Dynamical behavior [of mechanical systems] will be studied entirely in terms of arithmetic, or more precisely, in terms of high-speed arithmetic, for it is

\footnotetext{
${ }^{5}$ For details on Noether theorem, see (Butterfield 2006b).
} 
the availability of the modern digital computer which will make our approach both reasonable and practical. The dynamical equations of our models will be difference equations which, whether linear or nonlinear, well easily be solvable. Thereby, it is hoped that if an applied scientist is willing to learn the simple language of a computer, then he need be equipped only with the rudimentary mathematical knowledge of arithmetic and algebra in order to study highly complex physical phenomena. (Greenspan 1973, p. 4)

According to Greenspan, differential equations and real numbers can be eliminated from the study of mechanical systems. As the dynamics of mechanical systems is described by DM, its equations of motion are solved on computers, and as computers can only deal with natural and rational numbers, real numbers are not required to describe physical phenomena. Real numbers can be approximated on computers but this requires the use of rational or decimal numbers. Instead, one does not need this transformation in solving on computers the equations of DM formulated with rationals.

We disagree with Greenspan's evaluation of the capacities of his version of DM. By choosing to only use rational numbers, Greenspan severely restricts then expressive power of difference equations, for even though difference equations are by definition discrete, they may in general accept any real number as a solution (or any complex number in some cases). The restriction to rationals enables one to approximately solve Greenspan's DM equations on computers, but it is impossible or, at least, very difficult to solve them exactly or analytically without using the real numbers. Now, as we shall emphasize in section 2.3.2, exact results are extremely important in order to assess the performance of a physical theory.

Second, the only use of rational numbers may be not sufficient to describe physical phenomena even on computers. Greenspan himself acknowledges that, within his DM "classical continuous mathematics will be used [] in the study of stability, where properties of sets of rational numbers can be derived most easily by considering these numbers as subsets of the real number systems" (Greenspan 1973, p. 4). As a result, Greenspan's DM turns out to be unserviceable unless relying on real numbers. Thus, the development of Greenspan's DM does not imply that physics can dispense with the mathematical continuum for describing and explaining the behavior of mechanical systems.

\subsection{The continuous representation of time and dispensable representations}

Let us now present the answer we favor to the question of what the discrete representation of time in DM implies. According to this answer, the discrete representation of time in DM implies that physics can dispense with the continuous representation of time. In order to argue in favor of this claim, we first give some details about the conditions at which a physical theory can dispense with the continuous representation of time. The main condition is that the theory dispensing with the continuous representation of time should be usable by physicists, that is, it should enable physicists to describe, predict and explain physical phenomena. Second, we show that DM 
satisfies this condition and thus argue that the use of a discrete representation of time in DM implies that the continuous representation of time is dispensable in physics.

\subsubsection{Usable theories}

We begin our inquiry into the question whether the continuous representation of time is dispensable in physics by recalling that the notion of "dispensable" is stronger than the notion of "eliminable". In the original debate on the dispensability of various mathematical entities, a mathematical entity is said to be dispensable in a theory if it can be eliminated from the theory without any loss in its scope, the resulting theory being a good one (cf. Colyvan, 2001).

First, we suggest to extend the question of dispensability to mathematical representations, or mathematical tools of representation. The original debate focused on mathematical entities. However, in this paper, we are not interested in the question whether mathematical entities like numbers, sets, functions do exist but only in analyzing the notion of dispensability. There is no reason, in our view, to restrict this notion to entities.

Secondly, while "dispensability" is not "eliminability", it is unclear in the original debate, as Colyvan himself emphasizes, what a "good" theory is supposed to be. To remedy this deficiency, we suggest, that an entity or a mathematical representation can be said dispensable in a theory if it can be eliminated from the theory without any loss in its scope, the resulting theory being usable: it should be able to allow for description, prediction, and explanation of phenomena. Equipped with the notion of a usable theory, we can safely discard formal reconstructions of theories that (i) dispense with certain entities and (ii) are logically equivalent to the original ones, but (iii) cannot be used to investigate phenomena. These theories do not fall within the scope of the interesting theories for our purpose.

However, we reject Colyvan's criterion according to which the resulting theory should be preferable to the original theory, or at least more attractive. To our mind, it is not necessary to compare the resulting theory to the original one in order to decide whether an entity or a mathematical representation is dispensable in the former. The comparison constitutes a different, independent step in the analysis. For example, it seems that the notion of "force" can be eliminated from classical mechanics. Hamiltonian mechanics only seems to require the notion of "energy" as a fundamental concept (Wilson 2007, p. 175). If this is true, we would certainly be inclined to say that the notion of force is dispensable even if it is hard to say - and maybe not decidable - whether Newtonian or Hamiltonian mechanics is preferable. Maybe they are equally attractive or the one is preferable in some contexts and the other is preferable in other contexts. However, in our view, the notion of "force" is said dispensable in classical mechanics because it is eliminable and the resulting theory, Hamiltonian mechanics, is a usable theory.

\subsubsection{DM is a usable theory}

Let us now examine whether the version of DM we focus on in this paper can be said to be usable. Our main argument in favor of this claim is that the equations of motion 
for (at least some) mechanical systems can be given explicit solutions (D'Innocenzo et al. 1987). Explicit solutions are those expressed as functions of time. For instance, the equations of motion for a body subjected to a constant force and the harmonic oscillator system can be given explicit solutions. Let us briefly present these solutions (see Appendix 5.1 for more details).

Continuous Newtonian mechanics describes the phenomenon of falling bodies on Earth with the Newton's second law of motion, which is a vectorial differential equation:

$$
m d^{2} \mathbf{r} / d t^{2}=m \mathbf{g}
$$

The product of the mass by the acceleration on the left, where $\mathbf{r}$ is the position vector, is equal to the gravity force $m \mathbf{g}$ on the right. The $x$ and $z$ components of this vectorial equation have each an explicit solution, which are:

$$
x(t)=v_{x, i} t+x_{i} \text { and } z(t)=-1 / 2 g t^{2}+v_{z, i} t+z_{i}
$$

where $x$ and $z$ are the horizontal and vertical components, $x_{i}, z_{i}, v_{x, i}, v_{z, i}$ the initial conditions for position and speed, and $g$ the gravity constant. Similarly, in DM, we have, as explicit solutions for the two components:

$$
x_{k}=v_{x, i} t_{k}+x_{i} \text { and } z_{k}=-1 / 2 g t_{k}^{2}+\left(v_{z, i}+g h\right) t_{k}+z_{i}
$$

where there is in addition the discrete time step $h$. The harmonic oscillator can also be solved explicitly within DM. In that case, the solution is:

$$
x_{k}=A_{d} \cos \left(\omega_{d} t_{k}\right)+B_{d} \sin \left(\omega_{d} t_{k}\right)
$$

where $t_{k}$ is the discrete time, $A_{d}, B_{d}$ two constants depending on the discrete time step $h$, and $\omega_{d}$, the discrete pulsation also function of $h$. These two examples illustrate that some equations of motion can be solved explicitly within discrete mechanics.

The fact that some equations have explicit solutions within DM is remarkable because most equations of motion, both in continuous mechanics and in DM, do not have any explicit solutions. In continuous mechanics, the explicit solutions to most differential equations are indeed unknown or impossible to derive. However, even if only very few explicit solutions are known, scientists value their existence because an explicit solution provides them with a good understanding of the mechanical phenomenon. For instance, knowing the explicit solution of equation of motion of a falling body better enables one to grasp the phenomenon than only knowing a numerical solution on computer. That is because explicit solutions are both general and exact, which are two features that make them explanatory, as emphasized by Paul Humphreys:

The switch from analytic mathematics to numerical mathematics [...] has one immediate consequence: we often lose both the great generality and the potentially exact solutions that have traditionally been desired of scientific theories. (Humphreys 2004, p. 64) 
Let us review these two properties in turn.

- Generality: Explicit solutions are general in the sense that within them the position, as well as other variables, is a function of the relevant variables and parameters, thus requiring no specification of their numerical values. The explicit solutions express a relation between possibly all particular values of the parameters and variables of the function. For instance, in the case of the falling body problem, the solution $x_{k}$ represents the trajectory whatever the numerical values of the gravity constant $g$, or the initial conditions $x_{i}$ and $v_{i}$. It is a single formula describing a large range of different physical phenomena, for instance a falling body on Earth, or on the Moon, and with any value for its initial speed.

Generality, as a property of mathematical functions, is a key component in the explanatory role of explicit solutions. Explicit results provide explanations of physical phenomena in the sense that they show how the variations of some physical variables imply the variations of the other variables.

- Exactness: Explicit solutions being exact also accounts for their explanatory power. Exact solutions are such that, when put in place of the equations, they lead to an identity. For instance, $\sqrt{2}$ is an exact solution to the equation $x^{2}-2=0$ since, when put in place of $x$, the identity $0=0$ can be derived. In the same way, the explicit solutions of the falling body system with DM, equations (11) are exact solutions of the motion equations of DM (equations (18, 19) and 21, 22) since, put in place of them, they lead to the identity $1=1$.

Because there are exact, explicit solutions can be used to deduce other results about the system. Consequently, other features or properties of the system can be known and better understood. For instance, consider again the free falling body. As said above, the equations of motion for this system can be solved explicitly within DM: the positions $x_{k}$ and $z_{k}$ are functions of $t_{k}$. With these explicit solutions (11), it is first possible to derive the trajectory of the falling body. After some algebraic manipulations, the time $t_{k}$ is eliminated and the altitude $z_{k}$ is expressed as a function of the distance $x_{k}$ (see Appendix 5.2):

$$
z_{k}\left(x_{k}\right)=-\frac{g}{2} v_{x, i}^{2}\left(x_{k}-x_{i}\right)^{2}+\frac{v_{z, i}+g h}{v_{x, i}}\left(x_{k}-x_{i}\right)+z_{i}
$$

In this case, the deduced trajectory is a parabola. This provides a geometrical (vs. dynamical) representation of the phenomenon. It exhibits global information about the position of the falling body, enabling us to grasp the general behavior of falling bodies.

Second, specific, quantitative features of the trajectory can be deduced from the trajectory equation, increasing our knowledge of the system (for details, see Appendix 5.2. For instance, the highest position can be deduced via a few mathematical operations. It is the position where the partial derivative $z_{k}$ with respect to $x_{k}$ vanishes. It is reached at

$$
x_{k}^{*}=v_{x, i}\left(v_{z, i}+g h\right) / g+x_{i} \text { and } z_{k}^{*}=\left(v_{z, i}+g h\right)^{2} /(2 g)+z_{i}
$$

It is also possible to deduce the distance $x_{k}$ at which the falling body reaches the ground. Such deductions increase the explanatory power of the explicit solutions of the motion equations in enabling us to broaden our knowledge of the system. 
MD being able to provide explicit solutions to some mechanical problems is a strong argument to consider it as a usable theory. In particular, the existence of explicit solutions gives its principles the explanatory power that is expected from a usable physical theories. Needless to recall, MD being a discrete theory ensures that it can easily yield predictions, because it speaks the same language as computers.

Having argued that MD is a bona fide usable physical theory, we can claim that its existence, together with its predictive and explanatory powers, show that the continuous representation of time is dispensable in physical theories. In the next section, we discuss the implications of this claim.

\subsection{Dispensability and the structure of time}

Can we draw any conclusion about the structure of time from the dispensability argument we have just presented? Relying on existing discussions on this topic in the literature (Carnap 1966, Newton-Smith 1980, Maddy 1997), it seems that it should be so. In this section, we briefly review how this theme has been analyzed so far and present our own view.

The starting point of discussions about the relationship between the representation of time and the structure of time is the following observation. The use of the usual $t$ parameter in classical mechanics and other continuous theories is commonly associated, at least implicitly, with the assumption that the structure of time is continuous, in the following sense:

If time is continuous the set of all instants under the ordering given by being temporal before will be isomorphic to the reals or to some sub-sequence of the reals. (Newton-Smith 1980, p. 114)

This assumption seems to be generally accepted. For instance, in their textbook in classical mechanics, Kibble et al. adopt it as an explicit hypothesis of the theory (2004, p. 2). In Suppes' axiomatic reconstruction of classical mechanics, the statement according to which time is continuous (Suppes, 1999, p. 294) is presented as an axiom of the theory. In the chapter entitled "Indispensability and Scientific Practice" in her Naturalism in Mathematics, Maddy also asserts that the use of the real parameter $t$ in physical theories presupposes that time is continuous (1997, p. 155).

If one assumes that adopting the continuous representation of time is tantamount to hypothetizing that it has a continuous structure, as do Kibble et al., Maddy, NewtonSmith, and Suppes, it is clear that one should admit that the dispensability of the continuous representation has an impact on what is possible to claim about the structure of time. For instance, Newton-Smith claims, as far as we understand him, that the dispensability of the continuous representation of time implies that the structure of time is empirically undecidable (1980, p.126). More precisely, for the sake of his argument, he introduces two physical theories whose only difference resides in the way they represent time. The first one represents time by a parameter defined on real numbers and the second by a parameter defined on rationals. He shows that these theories are empirically equivalent. From this result, he concludes that the structure 
of time is empirically undecidable, thus relying on a property of the representation of time to argue about the structure of time

Can Newton-Smith' argument be transposed to DM so as to conclude that the dispensability of the continuous representation of time in DM leads to a similar undecidability result about the structure of time? Let us first emphasize that Newton-Smith does not distinguish between using the continuous representation of time and using real numbers in the representation of time. His inquiry focuses on the difference between reals and rationals as to their usefulness, or indispensability, in physics 7 On the contrary, as we have made explicit in section 1, DM being a discrete theory does not forbid it to use real numbers. This is a first important difference between the case imagined by Newton-Smith and the DM case, implying that Newton-Smith' argument cannot be transposed without important transformations to the DM case.

Admitting that we were willing to work out the relevant transformations, would it be appropriate to transpose Newton-Smith's argument? If it were the case, one could conclude from the study of DM that "time is continuous" is an undecidable statement. However, in our view, the very question whether "time is continuous" is undecidable is not legitimate. Let us make this point clear. Newton-Smith's argument in favour of the undecidability of the structure of time seems to presuppose that the way temporal parameters are used in physical theories puts constraints on the way we should conceive of the structure of time. Here is the answer Newton-Smith proposes to the question "why do we tend to regard time as continuous?":

[it] is quite simply that the best physical theories we have in fact constructed of the physical world require in their mathematical formulation a time parameter that ranges over the elements of the real number system. [...] We take individual real numbers as denoting instants and project back onto the interval a non-denumerably infinite number of extensionless parts or instants, one corresponding to each real number in the interval. That is, our belief in the continuity of time [...] arises from our projecting onto the world the richness that is present in the mathematical system which we have found to date to be essential to the construction of viable physical theories. (Newton-Smith, 1980, p. 118)

Consequently, according to Newton-Smith, "if our best theories should in the end turn out to involve representing time by a discrete time variable, we would have at

\footnotetext{
6 Newton-Smith then asks: "How ought we to respond to this undecidability result?"(1980, p. 126). According to him, the statement "time is continuous" can be interpreted as an empirical statement whose truth value is impossible to assess because there is no evidence to be had about it - this is "the Ignorance response". However, it can also be interpreted as a non-empirical statement describing a theoretical framework within which to describe empirical facts - this is "the Arrogance response". We do not discuss these interpretations since, as we make clear below in this Section, we deny that any conclusion about the undecidabilty of the structure of time can be drawn from physical theories, like DM, which represent time as discrete.

7 His line of inquiry thus continues Carnap's, who examined the following question: "Because irrational numbers are always the results of calculations, never the result of direct measurement, might it not be possible in physics to abandon irrational numbers altogether and work only with the rational numbers? That is certainly possible, but it would be a revolutionary change." (Carnap 1966, p. 88-89) As clear in the main text, this question is irrelevant to our own inquiry about the implications of the discrete representation of time in DM.
} 
least as much reason to regard time as discrete as we now have for regarding it as continuous"(ibid.). Here, we see that Newton-Smith is willing to read the structure of time off the physical theories. However, such an assumption is shaky; accordingly, we reject it. Indeed, we do not feel committed to regard time as "as a non-denumerably infinite number of extensionless parts or instant" even if physical theories represent time by the continuous parameter $t$. The reason why physical theories do so might be that they are not interested in investigating the structure of time and just use time as an element of the general framework in which the descriptions of phenomena hold.

Maddy also denies that the use of a continuous temporal parameter in physical theories commits us to claim that time is continuous. Her argument is slightly different. As we have seen above in the main text, she claims that representing time as continuous in physical theories amounts to hypothesizing that time is continuous. However, she argues that this hypothesis, and more generally "the physical structural assumptions underlying the application of the mathematics are not held to the same epistemic standard as ordinary physical assumptions" (Maddy 1997, p. 156). Even if the hypothesis of the continuity of time is actually made in physical theories, it is not endorsed by scientists. We agree with Maddy's conclusion. However, we reach it in a different way, by denying from the start that representing time by a continuous parameter amounts to hypothesizing that time is continuous. Accordingly, we claim, pace Newton-Smith, that the dispensability of the continuous representation of time is not sufficient to argue that the structure of time is undecidable.

At first glance, before any philosophical enquiry, the possible answers to the question whether time is continuous seem to be "yes" or "no". Thanks to Newton-Smith" work, it has been discovered that the answer may also be "undecidabe". In our view, the logical space is even more extended for there is a preliminary question to be asked: is the question legitimate? After having opened up the logical space, we close it by claiming that the question is illegitimate, thus making the former answers irrelevant. Our argument is that the use of a particular representation of time in physical theories (continuous, discrete or even another one) is not relevant to investigation on the structure time. Neither Carnap, Newton-Smith, or Maddy seems to consider the possibility we have mentioned. However, it allows one to understand why DM is viewed by its authors as having no implication whatsoever on an inquiry on the structure of time.

\subsection{Conclusion}

Our aim in this section has been to answer the question: What does it imply to use a discrete representation of time in physics? We have examined two answers that we found implausible. First, we have shown that adopting a discrete representation of time does not imply to use equations that are not as fundamental as continuous equations. In DM, the use of a discrete representation of time does not imply that discrete equations are model-specific and inevitably derived from fundamental, differential equations. In DM, the fundamental equations of motion are themselves discrete. Second, we have shown that the use of a discrete representation of time does not entail dispensing with the real numbers. For us, it is an important consequence. As far as we 
know, the distinction between representing time as continuous and using real numbers to represent time has not been introduced yet in the literature. We have made clear that in DM, time is represented as discrete even if instants are defined on real numbers.

We have then argued that an actual implication of the use of a discrete representation of time in physics is that physics can accordingly dispense with the continuous representation of time. In order to make explicit the meaning of this claim, we have first introduced the notion of a usable physical theory. It is a theory which enables one to actually describe, predict, and explain physical phenomena. Second, we have distinguished our dispensabilist implications from more ancient, similar claims, namely Carnap's and Newton-Smith. In our view, the question of the (continuous or discrete) structure of time cannot be legitimately asked when investigating how time is represented in (continuous and discrete) physical theories. We have suggested that the statement "time is continuous", besides being judged true, false, or undecidable, can also be judged irrelevant.

\section{Time symbols and their interpretation}

The aim of this section is to present another important feature of DM with respect to the representation of time, besides its being discrete. Within DM, the time symbol can be either a parameter, as it is the case in most physical theories, or a variable. This state of affair is distinctive of DM. We investigate its consequences. In order to do so, we first present how it comes that the time symbol is a dynamical variable in DM. In order to study what we can draw from this fact, we analyze the distinction between a variable and a parameter and how it applies to the time symbol. Finally, we discuss what difference it makes to treat the time symbol as a dynamical variable in a physical theory.

\subsection{The time symbol as a dynamical variable in DM}

In this section, we present the reason why the time symbol has to be considered a dynamical variable vs. a parameter in discrete mechanics. The parameter-variable distinction is not as obvious as it seems to be. A preliminary version of this distinction is that dynamical variables vary with respect to parameters, whereas parameters refer to the quantities with respect to which variables vary. In the following sections, we shall make this distinction more precise, but we begin with the presentation of the situation in DM.

To put it briefly, in DM, it is necessary to represent time as a dynamical variable unless energy conservation is lost. This is due to the Ge-Mardsen theorem whose content, in a nutshell, is the following. It states that when using discrete variational principles, that is, variational principles involving discrete time and space, it is impossible to have two desirable properties together if the time step is kept constant. 
These desirable properties are (i) the conservation of geometric properties, like symplecticity, and (ii) energy conservation ${ }^{8}$ (see Appendix 5.3 for more details).

So the Ge-Mardsen theorem seems to block up from the start the very possibility of building up satisfying discrete models - if time step is kept constant. Or, seen from the other side, the theorem entails that time-step adaptation (i.e. variability of the time-step) is needed to have symplectic-energy-momentum conservation. Thus, when one wants to preserve energy conservation in variational discrete models, the distribution of the time steps should be determined but by minimizing the discrete action. More precisely, as emphasized by D'Innocenzo et al. :

In the continuous case, energy conservation is a consequence of Newton's equations for conservative systems for which the Lagrangian does not depend explicitly on time. This is not automatic in an ad hoc discrete mechanics, on the contrary, only by treating both $\mathbf{r}$ and $\mathrm{t}$ as dynamical variables does one obtain both Newton's law and discrete energy conservation laws from the leastaction principle. Therefore the asymmetry present in continuous mechanics (where time is not treated on the same basis as the spatial coordinates) is eliminated. (D'Innocenzo et al. 1987, p. 246)

The sense in which time is represented by a discrete dynamical variable vs. a discrete parameter is thus that within DM, the time symbol is a variable with respect to which the action must be minimized. This means that its values are not arbitrary in the following sense: they are not determined by any external rule, like the rule $t_{k+1}=$ $t_{k}+h$ that is used in traditional discrete models such as models based on backward or forward Euler integrators (Section 1.1.1). The values of the time variable are instead determined by the evolution of the other variables.

Now that we have presented the reason why the time symbol is a discrete dynamical variable in DM, we turn back to the analysis of the variable-parameter distinction in the following section. This will allow us to draw some conclusion about the time symbol being a dynamical variable in DM.

\subsection{Variables and parameters}

In order to better capture the distinction between variables and parameters, we begin by briefly presenting a type of models in which the distinction is quite straightforward, namely causal models. Then we turn to the distinction as it holds in the general case. From this background, we move to the way time is represented in various physical theories. A last step will be necessary before examining further the status of the time symbol(s) in DM: we shall describe how time is represented in traditional discrete models.

\footnotetext{
8 "Given the importance of conserving integrals of motion and the important role played by the Hamiltonian structure in the reduction procedure for a system with symmetry, one might hope to find an algorithm that combines all of the desirable properties: conservation of energy, conservation of momenta (and other independent integrals), and conservation of the symplectic structure. However, one cannot do all three of these things at once unless one relaxes one or more of the conditions. [...]

It is interesting to note that when adaptive time steps are used, the arguments above no longer apply and indeed in this case it is possible to find integrators that are, in an appropriate sense, symplectic, energy preserving and momentum preserving, as shown in (Kane et al. 1999)." (Marsden 2009, p. 178).
} 


\subsubsection{Variables and parameters in scientific models}

Because the distinction between variables and parameters is difficult to capture, we first present a case in which it is reasonably clear.

The distinction between variables and parameters is well defined only in the context of a given model. It is largely a matter of the aims of modelling, a consequence of the somewhat arbitrary but unavoidable division between what is endogenous and what is left exogenous and what is considered as changeable and what fixed. Causal relations are change relating and what are perceived to be the relevant causal relations depends, to some extent, on what kinds of possible changes are thought to be relevant. If the modeller is interested in how some features of a system are determined by other features of a system, she endogenizes these features as dependent variables. If some features are taken for granted or possible changes in these features are thought to be unlikely or irrelevant, they are left as parameters (Kuorikoski, 2012, p. 368)

In causal models, a parameter thus describes the background against which variables vary, whereas variables are used to represent the causal relations one is interested in. It may be noted that according to the model's purpose, a quantity may be sometimes represented as a variable, and by a parameter when the modelers is interested in another question.

Let us now turn to physical models. Not all physical theories represent causal relationships; however, the distinction between variables and parameters still holds in non-causal theories, with the same general meaning. It reflects the different ways the represented quantities may depend on each other. A variable can depend on the variations of a parameter, whereas a parameter has its own source of variation, which is independent of the variation of the other represented quantities.

To sum up, a variable is a symbol of a magnitude whose variations are the topic of the formulae; a parameter helps formulae to say something about the variations of variables.

\subsubsection{The case of the time symbol}

Equipped with this understanding of the distinction between variables and parameters, we now turn to the fate of the time symbol in physical theories, where it is a parameter more often than a variable. For instance, the time symbol is a parameter in classical mechanics, statistical mechanics, quantum mechanics, in hydrodynamics, electromagnetism. As these theories describe the evolution of physical phenomena over time, time is in the background, so to speak. It is not an object of investigation in itself. By contrast, in cosmology, the time symbol can be a variable: it can vary with the scale factor or with temperature. Thus, in contemporary physical theories, there is no uniform representation of time: it is usually a parameter, but it is a variable in some theories.

The general situation in physics is that the physical theories do not have anything to say about time proper. They just use it as an element of the general framework in which the descriptions of phenomena hold. The symbol $t$ has a life of its own in the 
equations, so to speak. It is independent of the physical laws in the sense that it does not enter into the equations otherwise than in "going further", or "stepping further", that is, in going from one instant to the next.

Two remarks may complement this brief description. (i) Things were different at the beginning of physical theorizing. As is well-known, Newton devoted a few pages of the Principia to a discourse about space and time. In his view, a physical theory like his own had to say something about space and time: he conceived space and time as proper objects of physical theorizing and his discourse about them as a necessary component of his theory. (ii) Within modern theories, fragmented as they are, it is striking that the theories which represent time by a parameter are deliberately at odds with the theories that say something about time. For instance, in classical mechanics as well as in non-relativistic quantum theory, time is represented as entirely separated from space. It is as if these theories did not take seriously what relativity (special and general) has to say about the nature of time. For sure, the formal and empirical relationships among these theories are relatively clear and the separation of space and time in non-relativistic theories does not raise any problem of consistency, let alone empirical problems. However, it is notoriously difficult to translate these mathematical relationships into conceptual relationships. Even though the intertheoretical relationships are well-controlled, this does not imply that the conceptual relationships are as controlled. There is a sort of blind spot here: the concept of time in classical mechanics, say, is clearly different from the concept of time in relativity, and the difference cannot be reduced to any approximation relation.

To sum up, there is no uniform representation of time in physical theories, past or modern. The representation of time is even so diverse that it is difficult to articulate the roles played by the symbols representing time in the various theories.

\subsubsection{The time symbol( $(s)$ in discrete models}

In order to complete our review of the various ways time is represented in physics, we now inquire into the way time is represented in traditional discrete models, that is, discrete models not relying on DM.

In traditional discrete models, the discrete symbol $t_{k}$ is always a parameter in the sense that it is not an object of investigation but rather a vehicle of the investigation. The discrete variables evolve from one $t_{k}$ to $t_{k}+h$, etc. In these models, "stepping further" means going from one instant to the nex $9^{9}$ The main difference with continuous models lies in the fact that the set of possible values for tk is discrete rather than continuous 10

There is another difference, however. Contrary to the continuous time-symbol $t$, the time-symbol $t_{k}$ is associated to two different temporal notions, namely $t_{k}$ and $k$.

\footnotetext{
9 There are further aspects to the use of time symbols in discrete models. On the one hand, there are numerical methods with "stage" like the Runge-Kutta methods. Here, intermediate discrete instants $t_{k, i}$, where $i$ is an integer, are introduced between two consecutive instants $t_{k}$ and $t_{k}+h$. Two levels of time discretization are thus used. On the other hand, there are discrete models with adaptive discrete time symbols: the time step between two consecutive discrete time symbols $t_{k}$ and $t_{k+1}$ is variable. At each discrete instant, the time step is adapted according to criteria of computational accuracy.

10 All these notions differ from "the time of the simulation", i.e., the time spent by the computer to solve the discrete model's equations. In the remaining of the paper, we shall put this notion aside.
} 
The discrete time-symbol $t_{k}$ plays the same role as the continuous time-symbol $t$ : it represents the time over which phenomena occur. $k$, on the other hand, represents the "time in the model", so to speak. It represents the time over which the representations of the phenomena occur. The rule " $k \rightarrow k+1$ ", by which $k$ is "stepping further", governs the evolution of symbol $t_{k}$ in traditional discrete models.

Let us take the example of a free particle. With a continuous model, its motion can be described by the equation $x=v_{i} t+x_{i}$, where $x$ represents the position of the particle, $v_{i}$ and $x_{i}$ the initial conditions, and $t$ the time over which the particle is moving. Within a discrete model, the motion of the particle can be described by the equation $x_{k}=v_{i} t_{k}+x_{i}$. Like $t, t_{k}$ represents the time over which the particle is moving. It can be expressed in seconds, minutes, days etc. depending on the phenomena. $t_{k}$ is defined as the product of $h$ by $k$, where $h$ is the discrete time step and $k$, an integer. $k$ is a mathematical parameter that marks the discrete instants; it does not represent the time over which phenomena happen. However, the increment " +1 " in the passage from $k$ to $k+1$ determines the evolution of discrete time from the instant $t_{k}$ to its successor $t_{k+1}$. Depending of the size of the time step $h$, the same increment " +1 " corresponds to different evolutions of the time parameter $t_{k}$, e.g., one micro-second, one second, one day etc. Thus, because the rule " $k \rightarrow k+1$ " governs the evolution of the discrete time $t_{k}$, we call it the "time of the model". It is the clock, so to speak, that makes the discrete time parameter $t_{k}$ evolve ${ }^{11}$. In the following section, we shall investigate the fate of both temporal notions in the framework of DM.

\subsubsection{The time symbol in DM}

In order to analyze the time symbol $t_{k}$ as it is used in DM, it seems to us useful to introduce some distinctions concerning the temporal notions that are at play in physical theories and models. They are meant to summarize and develop the abovementioned points about time symbols.

(i) We first have to mention the ordinary time, that is, the time in which the investigated phenomena take place, or the time over which the states of the investigated systems change. Let us name it "time*".

(ii) In physics, time* is represented by a time-symbol written either $t$, or, in discrete models and DMs, $t_{k}$. As emphasized about, $t$ and $t_{k}$ are often parameters.

(iii) It is also important to include the "time of the model" in the discussion, i.e., the time over which the representations of the phenomena change, from one state to another. In continuous models and theories, there is not difference between the representation of time* and the time of the model: the same symbol $t$ holds for both.

\footnotetext{
11 We interpret $k$ in a temporal way, as the "time of the model". However, it seems that one might also interpret it in a spatial way since the change " $k \rightarrow k+1$ " governs also the evolution of the position $x_{k}$ to $x_{k+1}$. Nevertheless, at least within DM, $k$ seems to be more a temporal parameter than a spatial one. One reason would be that, to our knowledge, the parameter $k$ shares with $t_{k}$ an order property: when $k<k+1$ one cannot have $t_{k}>t_{k+1}$. In other words, $k$ and $t_{k}$ have the same direction. On contrary, while $k<k+1$, one can have $x_{k}<x_{k+1}$ as well as $x_{k}>x_{k+1}$. Besides, when discrete equations are implemented on computers, there might be other kinds of "clocks". For example, the computation of the coordinates $\left(x_{k+1}, t_{k+1}\right)$ might be implemented in a device before the computation of the coordinates $\left(x_{k}, t_{k}\right)$. There would be a clock that commands to use $k+1$ before $k$. Since we do not discuss implementation issues and focus on the equations of DM, we leave aside this point.
} 
In discrete models and in DM, by contrast, it is noted $k, k$ being an integer. $k$ cannot be but a parameter.

The main difference between DM and other discrete models is that in DM, the way the "time of the model" goes from $t_{k}$ to $t_{k+1}$ is determined by the equations of the investigated system rather than by a rule that is unconnected to the system's equations. Therefore, $t_{k}$ is a variable relative to the parameter $k$. As a result, the representation of time has a dual nature in DM: whereas time* is represented by $t_{k}$, the "time of the model" is represented by $k$. This builds up a sharp contrast with both continuous models and traditional discrete models.

\subsection{Discussion}

Let us now to analyze the implication of the dual representation of time in DM. We first examine whether it is indispensable in DM. Then we introduce a new distinction within the notion of representation, from which we argue that the indispensability of the dual representation of time may be interpreted in a deflationary fashion.

\subsubsection{Is the dual representation of time indispensable in DM?}

How are we to interpret the difference between $k$ and $t_{k}$ within DM? Two options are available. The first one is to claim that the difference between $k$ and $t_{k}$ is a necessary feature of DM and cannot be dispensed with. This would imply that discrete mechanics allows for new insights about time: according to this interpretation, the fact that $t_{k}$ varies with the other physical variables says something about the nature of time*. The second option is to consider the difference between $k$ and $t_{k}$ as an artefact of DM.

The second option looks disappointing because it does not do justice to the fact that within DM, $t_{k}$ actually depends on the system's dynamics. In order to consider this as an artefact of the representation being discrete, some explanation has to be given why and how an artefact could transform a symbol that is very commonly a parameter into a variable. Now, this explanation looks difficult to find out. Accordingly, in the following, we focus on the first option.

\subsubsection{Representing by vs. representing as}

According to the first above-presented option, the difference between $k$ and $t_{k}$ is a necessary feature of DM, which seems to imply that DM has something new to say about time*. To what extent is this implication sound? In this section, we investigate whether adopting the first option necessarily involves jumping to the conclusion that DM says something new about the structure of time*.

In order to do so, let us introduce a distinction within the concept of representation. In what follows, we shall call "a representation" any concrete item (like ink imprints) that is used as a representation by an agent, and understood as such by another agent, the receiver. According to this definition, a representation relates four items: the user of the representation, its content, the receiver, and the medium of the representation (e.g., line-drawing, painting, newspaper article, etc.) Here, we focus on 


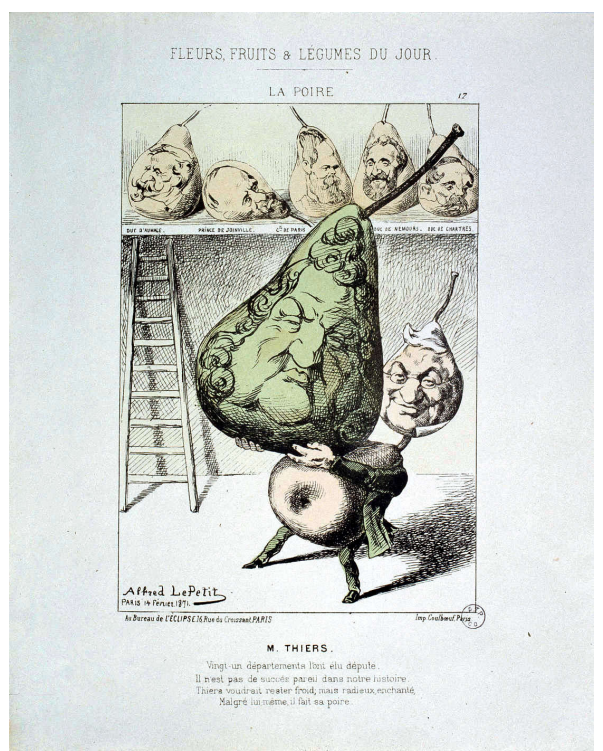

Fig. 3 "Fleurs, fruits et légumes du jour - La poire - M. Thiers", Alfred Le Petit (1871). C Picture RMNGrand Palais - M. Bellot.

the relationship between the content of the representation and the medium. In some cases, this relationship is based on physical interactions, like in photographs, but in many cases, it is only based on conventions. For instance, one may decide that this pencil represents a railway. In this case, the railway is represented by the pencil. The pencil, associated with the appropriate convention, is the medium of the representation. However, the medium of the representation may also play another role. Let us take the classical example of the French politician Thiers represented as a pear (see Fig. (37). In this caricature, Thiers is not represented by a pear but as a pear. This difference may be subtle, but it is important to understand it in order to rightly interpret the meaning of representations. If someone understood the caricature of Thiers as meaning that he is a gardener, it would be a gross error ${ }^{12}$ The difference between "represented by" and "represented as" is central in the way we use pictures and symbols to communicate and share meanings.

Prima facie, the difference between "represented by" and "represented as" seems relevant to the interpretation of symbols in physics. Let us now suggest how this distinction is usually applied to the symbols that are usually used in physics.

On the one hand, when the physical quantity $\mathrm{X}$ is represented by the symbol $x$, the symbol $x$ holds for $\mathrm{X}$ in the picture provided by the theory in which $x$ is used. " $x$ " is not only a letter; in physics, it is always presupposed to be the name of a variable, taking its values within this or that set of numbers (the integers, the reals, or a discrete set of the reals, etc.). The set may be chosen because it is supposed to be faithful to the nature of $\mathrm{X}$, or for commodity reasons. As an example of the second case, $\mathrm{X}$ can

\footnotetext{
12 In the $19^{\text {th }}$ century, pear is known in France to be a metaphor for bourgeois monarchy. This caricature shows thus the affinity of Thiers with this political regime.
} 
be represented by a symbol taking its value in a discrete (resp. continuous) set of numbers without any implication on the discrete (resp. continuous) nature of $\mathrm{X}$.

On the other hand, representing a physical quantity $\mathrm{X}$ as an $\mathrm{A}$ or a B, for instance, as a variable or as a parameter, involves interpreting $\mathrm{X}$ as endowed with the properties of the As or Bs. For instance, representing time as a variable involves interpreting time as dependent of other variables, whereas interpreting it as a parameter involves interpreting it as independent of other variables. Representing $\mathrm{X}$ as an $\mathrm{A}$ or a $\mathrm{B}$ thus involves a (more or less implicit) a claim about the nature of $\mathrm{X}$.

\subsubsection{Representing by, representing as, variables, and parameters}

The above examples seem to imply that physical quantities are represented as variables or parameters. This seems to be the common way to use the variable-parameter distinction. In this last section, we want to suggest that it is also possible to interpret "variable" and "parameter" as naming different mediums of representation rather than as saying anything about the nature of the represented quantities. Accordingly, we claim that some physical quantities can be represented by variables or parameters, in the very same way as they can be represented by real numbers.

Let us now present the implications of our claim. According to it, representing a physical quantity by a variable does not involve any claim about its nature. It does not involve that it is intrinsically linked to other variables, for instance. To put it in other words, in our view, variables and parameters are just representational tools, deprived of any further content or presupposition. In particular, $t_{k}$ being a variable in $\mathrm{DM}$, whereas $k$ is a parameter whose role is similar to the role of the usual continuous parameter $t$ in continuous theory, does not imply that DM reveals that time* is endowed with a double nature.

We do not generalize our conclusion from the variable $t_{k}$ in DM to other variables and parameters in physical theories. As already discussed in the Section 2.4, time plays a special role in physical theories. They do not generally investigate time. That is why we claim that time should be viewed as represented by rather represented as variables and parameters. However, we do not extend this claim to other physical magnitudes.

To put it in a nutshell, our claim is that the indispensable, dual representation of time* in DM has not implication about the nature of time*. Indispensability is no guide toward ontological conclusions, but rather a guide toward a complete analysis of the means we use to represent quantities in physics.

\subsection{Conclusion}

In this section, we have first presented the reasons why $t_{k}$ can be said to be a dynamical variable within DM, whereas $t$ is usually a parameter in continuous physical theories. In order to better appreciate the meaning of $t_{k}$ being a dynamical variable, we have then discussed the distinction between variables and parameter as it is used in empirical sciences. At last, we have argued that the dual representation of time in DM, even though it is indispensable, has no ontological implications if one accepts 
to consider that it is possible to represent a physical quantity by a variable (resp. a parameter) instead of representing it as a variable (resp. a parameter).

\section{Concluding remarks}

Our aim in this paper has been to investigate into the consequences of adopting a discrete physical theory on the representation of time. By doing so we have found out that the fundamental opposition between a continuous and a discrete representation has to be supplemented with three other oppositions.

The first one is the opposition between a discrete representation only using natural and rational numbers (as focused on by Carnap and Newton-Smith) and a discrete representation by means of discrete sets of real numbers, as currently used in DM. The availability of the second type of discreteness radically changes the terms of the old discussion about the dispensability of the continuous representation of time, as we have shown in section 2. In particular, we have argued that one can dispense with the continuous representation of time in physical theories. However, we have emphasized that one should not draw any consequence from this result on the structure of time. "Is the structure of time continuous?" is sometimes an irrelevant question when one focuses on how time is represented in physical theories.

The second opposition is between variables and parameters. This opposition is widely used, but its interpretation varies from one domain to the other. We have tried to make clear its meaning in physical theories in section 3 .

The third opposition we have uncovered is between two sorts of representing, namely representing by and representing as. It is commonly assumed that when a physical quantity is represented by a discrete symbol, i.e., a symbol taking its value in a discrete set of numbers, there is no reason to interpret such a use as meaning that the quantity is conceived of as discrete. Representing by a discrete symbol does not indicate any wish to foster any metaphysical assumption. By contrast, it is commonly assumed that representing a physical quantity as a dynamical variable implies that this quantity plays a certain role among other relevant quantities. Representing as a dynamical variable fosters substantial assumptions about the quantity so represented. In section 3, we have shown that these common assumptions are misleading. The current state of DM induces us to claim that there is no reason to associate substantial assumptions with the act of representing as a variable or a parameter. The variable/parameter opposition should be viewed as of the same type as the continuous/discrete opposition: it does not involve any interpretation advice. Accordingly, it is more appropriate to use the expression "representing by a variable (resp. a parameter)" instead of "representing as variable (resp. a parameter)".

The conclusion we have just summed up not only applies to DM, but virtually to every other physical theories as well, even continuous ones. It sheds light on the richness of the practices of representing time in physics and helps distinguish between aspects that only pertain to representational skills and aspects that are linked with scientific hypotheses about time. 
Acknowledgements We wish to thank the participants of the conference Time of Nature, Nature of Time for comments and discussion. We are most grateful to Christophe Bouton and Philippe Huneman for helpful comments on previous drafts of this paper. We would like also to thank Paul Humphreys for precious suggestions and remarks on the paper.

\section{Appendix}

5.1 Discrete Mechanics and some applications

Let us call $A_{d}$ the action in Discrete Mechanics (for details see (D'Innocenzo et al. 1987; Marsden et al. 2001, chapter 4)):

$$
A_{d}=\sum_{k=0}^{N-1} L_{d} \cdot\left(t_{k+1}-t_{k}\right)
$$

with $L_{d}$ the discrete Lagrangian. The principle of least action states that : $\delta A_{d}=0$. It results the following twofold discrete Euler-Lagrange equations (DEL). The first discrete Euler-Lagrange equation is:

$$
\left(t_{k}-t_{k-1}\right) \frac{\partial L_{d}\left(t_{k-1}, q_{k-1}, t_{k}, q_{k}\right)}{\partial q_{k}}+\left(t_{k+1}-t_{k}\right) \frac{\partial L_{d}\left(t_{k}, q_{k}, t_{k+1}, q_{k+1}\right)}{\partial q_{k}}=0
$$

The second discrete Euler-Lagrange equation is:

$$
\frac{\partial}{\partial t_{k}}\left[\left(t_{k}-t_{k-1}\right) L_{d}\left(t_{k-1}, q_{k-1}, t_{k}, q_{k}\right)\right]+\frac{\partial}{\partial t_{k}}\left[\left(t_{k+1}-t_{k}\right) L_{d}\left(t_{k}, q_{k}, t_{k+1}, q_{k+1}\right)\right]=0
$$

Let us follow d'Innocenzo et al. for the choice of the discrete Lagrangian :

$$
L_{d}\left(q_{k}, q_{k+1}, h_{k+1}\right)=\frac{1}{2} m\left(\frac{q_{k+1}-q_{k}}{h_{k+1}}\right)^{2}-V\left(\frac{q_{k+1}+q_{k}}{2}\right)
$$

with $h_{k+1}=t_{k+1}-t_{k}$. Under these conditions, let us solve (i) the free particle system, (ii) the one dimension falling body problem and (iii) the one dimension harmonic oscillator system.

(i) The free particle is the system with $V=V_{0}$. The DEL become:

$$
\begin{aligned}
& m \frac{v_{x, k+1}-v_{x, k}}{h_{k+1}}=0 \\
& \frac{1}{2} m v_{x, k+1}^{2}=\frac{1}{2} m v_{x, k}^{2}
\end{aligned}
$$

with $v_{x, k}=\frac{x_{k}-x_{k-1}}{t_{k}-t_{k-1}}$. The solution of the equations is:

$$
x_{k}=v_{x, i} t_{k}+x_{i}
$$

with the initial conditions $t_{0}=0, x_{i}=x_{0}, v_{x, i}=\frac{x_{1}-x_{0}}{h_{1}}$. 
(ii) The one dimension falling body problem is the system where $V\left(z_{k}, z_{k+1}\right)=$ $m g \frac{z_{k+1}+z_{k}}{2}$. Thus, the DEL are :

$$
\begin{aligned}
& v_{z, k+1}-v_{z, k}=-\frac{g}{2}\left(t_{k+1}-t_{k-1}\right) \\
& \frac{1}{2} m v_{z, k+1}^{2}+m g \frac{z_{k+1}+z_{k}}{2}=\frac{1}{2} m v_{z, k}^{2}+m g \frac{z_{k}+z_{k-1}}{2}
\end{aligned}
$$

with $v_{z, k}=\frac{z_{k}-z_{k-1}}{t_{k}-t_{k-1}}$. I follow the resolution of d'Innocenzo et al. (1987) with different notations. The solution of the equations is $t_{k}=h$ and :

$$
z_{k}=-\frac{g}{2} t_{k}^{2}+\left(v_{z, i}+g h\right) t_{k}+z_{i}
$$

with the initial conditions $t_{0}=0, z_{i}=z_{0} v_{z, i}=\frac{z_{1}-z_{0}}{h_{1}}$.

(iii) The one dimension harmonic oscillator system is the system where $V\left(x_{k}, x_{k+1}\right)=$ $\frac{1}{2} K\left(\frac{x_{k+1}+x_{k}}{2}\right)^{2}$. Thus, the DEL are:

$$
\begin{aligned}
& m\left(v_{x, k+1}-v_{x, k}\right)=-\frac{K}{4}\left[\left(x_{k}+x_{k-1}\right) h_{k}+\left(x_{k+1}+x_{k}\right) h_{k+1}\right] \\
& \frac{1}{2} m v_{x, k+1}^{2}+\frac{1}{2} K\left(\frac{x_{k+1}+x_{k}}{2}\right)^{2}=\frac{1}{2} m v_{x, k}^{2}+\frac{1}{2} K\left(\frac{x_{k}+x_{k-1}}{2}\right)^{2}
\end{aligned}
$$

Following d'Innocenzo et al. (1987) with different notations (in particular see (Cieslinski \& Ratkiewicz 2006)), we have:

$$
x_{k}=x_{0} \cos \left(\omega_{d} t_{k}\right)+\frac{x_{1}-x_{0} \cos \left(\omega_{d} h\right)}{\sin \left(\omega_{d} h\right)} \sin \left(\omega_{d} t_{k}\right)
$$

with $\omega_{d}=\frac{1}{h} \arctan \left(\frac{\omega h}{1-\omega^{2} h^{2} / 4}\right)$ and $\omega=\sqrt{\frac{K}{m}}$.

\subsection{Trajectory of a falling body in DM}

Since $x_{k}=v_{x, i} t_{k}+x_{0}$ (see equation 201 in Appendix) then, $t_{k}=\left(x_{k}-x_{i}\right) / v_{x, i}$. Hence, put it in the equation (23) in Appendix, we derive the equation of the trajectory :

$$
\begin{aligned}
z_{k} & =-\frac{g}{2} t_{k}^{2}+\left(v_{z, i}+g h\right) t_{k}+z_{i} \\
& =-\frac{g}{2 v_{x, i}^{2}}\left(x_{k}-x_{0}\right)^{2}+\frac{v_{z, i}+g h}{v_{x, i}}\left(x_{k}-x_{i}\right)+z_{i}
\end{aligned}
$$

Now, we can deduce the highest position of the body. It is the position where the partial derivative $z_{k}$ with respect to $x_{k}$ vanishes : $\frac{\partial z_{k}}{\partial x_{k}}\left(x_{k}^{*}\right)=0$. Hence,

$$
\begin{aligned}
& x_{k}^{*}=\frac{v_{x, i}\left(v_{z, i}+g h\right)}{g}+x_{i} \\
& z_{k}^{*}=z_{k}\left(x_{k}^{*}\right)=\frac{\left(v_{z, i}+g h\right)^{2}}{2 g}+z_{i}
\end{aligned}
$$




\subsection{Ge-Marsden Theorem}

We report here the Ge-Marsden theorem and its proof as they are formulated in the original paper:

We recall that there are algorithms which exactly preserve energy, some of which also preserve other quantities [...]. However, these algorithms cannot be symplectic, according to the following result of $\mathrm{Ge}$ :

Let $H$ be a Hamiltonian which has no conserved quantities (in a given class $\mathscr{H}$, for example analytic functions) other than functions of $H$. That is, if $\{F, H\}=0$, then $F(z)=F_{0}(H(z))$ for a function $F_{0}$. Let $\Phi_{\Delta t}$ be an algorithm which is defined for small $\Delta t$ and is smooth. If this algorithm is symplectic, and conserved $H$ exactly, then it is the time advance map for the exact Hamiltonian system up to a reparametrization of time. In other words, approximate symplectic algorithms cannot preserve energy for nonintegrable systems.

This result is in fact easy to prove. The algorithm being symplectic, is generated by a dependent function $F(z, t)$, which we assume belong to $\mathscr{H}$. Since $\Phi_{\Delta t}$ preserves $H$, and $H$ is assume to be time independent, $F$ commutes with $H$, and so $F(z)=F_{0}(H(z))$. It follows that the hamiltonian vector fields of $F$ and $H$ are parallel, so their integral curves are related by a time reparametrization. (Ge \& Marsden 1988, p. 135).

As far we understand this result, while the Hamiltonian system is assumed to be nonintegrable - i.e that exact solution cannot be constructed - if the symplectic algorithm exactly preserves energy, the solution of the algorithm would be the exact solution of the system modulo a reparametrization. This contradicts the assumption according to which the system is nonintegrable (see also (Ge 1991, p. 380; Marsden 2009, p. 178)). However, as Marsden (2009, p. 179) emphasizes, if the time step is variable, the previous result does not hold and symplectic algorithms can exactly preserve energy.

\section{References}

Butterfield, J. (2006a). On Symplectic Reduction in Classical Mechanics, in Earman, J. \& Butterfield, J. (eds.). The Handbook of Philosophy of Physics, North Holland: Elsevier, 1-131.

Butterfield, J. (2006b). On Symmetries and Conserved Quantities in Classical Mechanics, in Demopoulos, W. \& Pitowsky, I. (eds.). Physical Theory and its Interpretation, Dordrecht: Springer 2006, 43-99.

Carnap, R. (1966). Philosophical Foundations of Physics. New York and London: Basic Books.

Cieslinski, J. L. \& Ratkiewicz, B. (2006). On Simulations of the Classical Harmonic Oscillator Equation by Difference Equations, Advances in Difference Equations, p. 1-17.

Colyvan, M. (2001). The Indispensability of Mathematics. Oxford: Oxford University Press.

D'Innocenzo, A., Renna, L. \& Rotelli, P. (1987). Some Studies in Discrete Mechanics. European Journal of Physics, 8, 245-252.

Feng, K. \& Qin, M. (2010). Symplectic Geometric Algorithms for Hamiltonian Systems, Heidelberg, Dordrecht, London, New-York : Springer.

Ge, Z. \& Marsden, J. E. (1988). Lie-Poisson Hamilton-Jacobi Theory and Lie-Poisson Integrators, Physics Letters A, 133 (3), 134-139.

Ge, Z. (1991). Equivariant Symplectic Difference Schemes and Generating Functions, Physica D, 49, p. 376-386.

Greenspan, D. (1973). Discrete Models. Addison-Wesley Publishing Company. 
Hairer E., Lubich, C. and Wanner, G. (2006). Geometric Numerical Integration: Structure-Preserving Algorithms for Ordinary Differential Equations, Berlin, Heidelberg: Springer.

Humphreys, P. (2004). Extending Ourselves: Computational Science, Empiricism, and Scientific Method, New-York: Oxford University Press.

Kane, C., Marsden, J. E. \& Ortiz, M. (1999). Symplectic-Energy-Momentum Preserving Variational Integrators, Journal of Mathematical Physics, 40, p. 3353-3371.

Kibble, Tom W. B. \& Berkshire, Frank H. (2009). Classical Mechanics, London: Imperial College Press. Kuorikoski, J. (2012). Mechanisms, Modularity and Constitutive Explanation, Erkenntnis, 77 (3), 361 380 .

LaBudde, R. A. \& Greenspan, D. (1974). Discrete Mechanics-A General Treatment. Journal of Computational Physics, 15, 134-167.

Lee, T. D. (1983). Can Time Be a Discrete Dynamical Variable?. Physics Letters, 122B (3-4), 217-220.

Lee, T.D. (1987). Difference Equations and Conservation Laws. Journal of Statistical Physics, 46 (5-6), 843-860.

Lee, T., Leok, M. \& McClamroch, H. (2009). Discrete Control Systems. In Robert A. Meyer (Ed.). Encyclopedia of Complexity and Systems Science (pp. 2002-2019). New York:Springer.

Maddy, P. (1997). Naturalism in Mathematics. Oxford: Clarendon Press.

Marsden, J. E. \& West M. (2001). Discrete Mechanics and Variational Integrators. Acta Numerica, 10, 357-514.

Marsden, J. E. (2009). Lectures on Mechanics. Cambridge: Cambridge University Press.

Newton-Smith, W. H. (1980). The Structure of Time. London: Routledge \& Kegan Paul.

North, J. (2009). The Structure of Physics: A Case Study, Journal of Philosophy, 106 (2), 57-88.

Stern, A. \& Desbrun, M. (2008). Discrete Geometric Mechanics for variational Time Integrators, in

Discrete Differential Geometry: An Applied Introduction, Siggraph 2006 Course Notes, chap. 15.

Suppes, P. (1957). Introduction to Logic. New York: Van Nostrand Reinhold.

Wilson, J. (2007). Newtonian Forces. The British Journal for Philosophy of Science, 58, p. 173-205. 